Cyril Fernando Memorial Oration 2005

\title{
Guillain-Barre syndrome - a Sri Lankan profile
}

\author{
Ranawaka U K ${ }^{1}$ \\ Journal of the Ceylon College of Physicians, 2014, 45, 6-15
}

\section{Introduction}

Guillain-Barre syndrome (GBS) is a devastating neurological illness, which leads to significant mortality and disability. Its impact is even more serious when considering the fact that most affected individuals are young adults in the prime of their lives. Prompt diagnosis is important as early treatment improves outcome. There has been a resurgence of interest in GBS with the identification of its neurophysiological and pathological subtypes, demonstration of its association with Campylobacter jejuni enteritis, and discovery of associated anti-ganglioside antibodies which have some correlation with the disease.

GBS is an acute inflammatory poly-radiculoneuropathy. It is characterised by acute, hyporeflexic or areflexic, flaccid paralysis. Weakness commonly starts in the lower limbs, but rapidly spreads to involve the upper limbs, and sometimes, the cranial nerves. Sensory symptoms are common, but objective signs unusual. Up to two-thirds of patients have a history of preceding illness, usually a respiratory infection or diarrhoeal illness.

The illness progresses rapidly for a period of up to 4 weeks, followed by a plateau phase of 2-4 weeks, before recovery starts. Death during the acute phase can occur due to involvement of respiratory muscles leading to neuromuscular respiratory failure, aspiration pneumonia, and cardiac autonomic disturbances. Recovery is seldom complete. At one year after the illness, $5-10 \%$ of patients would be dead, and $30-40 \%$ have residual disability. Only about $50-70 \%$ are asymptomatic or are left with only minor symptoms not interfering with their daily lives.

The clinical picture of 'classical' Guillain-Barre syndrome, as described above, is similar throughout the world.1-10 GBS, however, is pathologically heterogeneous, with the underlying pathological process being demyelination or axonal degeneration.

GBS is an immune mediated disease. The antecedent infection triggers off an immune response which causes damage to neural tissue due to

\footnotetext{
${ }^{1}$ Consultant Neurologist and Senior Lecturer in Medicine, Faculty of Medicine, University of Kelaniya, Sri Lanka.
}

'molecular mimicry', the presence of identical antigenic epitopes on the pathogen and the peripheral nerves. When these 'shared epitopes' are present on the myelin sheath or the Schwann cells of the peripheral nerves, the immune response damages the myelin, leading to demyelination. When the antigenic epitopes are located on the axonal membrane of the nerves, axonal degeneration results. The pathological type can be neurophysiologically distinguished, enabling classification of GBS into acute inflammatory demyelinating polyradiculoneuropathy (AIDP) or axonal GBS. Axonal GBS is further subdivided into acute motor axonal neuropathy (AMAN) or acute motor-sensory axonal neuropathy (AMSAN), depending on the presence of sensory fibre involvement.

The pathological and neurophysiological type seems to vary according to the geographical location. In North America and Europe, AIDP constitutes $56-87 \%$ of GBS cases, and axonal GBS accounts for $3-10 \% .^{2,11-14}$ In contrast, axonal GBS is commoner in the Far Eastern countries such as China and Japan, ranging from $33 \%$ to $98 \% .^{10,15-22}$ Such distinctions are not merely academic, as the underlying aetiology and the outcome appear to be different. Axonal GBS, especially AMAN in Far Eastern countries, is associated with Campylobacter jejuni infection. ${ }^{12,15,23,24}$ AMSAN in Western countries is associated with a poor outcome, whereas AMAN in Chinese children is associated with a good prognosis. ${ }^{15,16,23-25}$

Similar data from South Asian countries, especially the Indian subcontinent, are limited. There are no published prospective data on the clinical and electrophysiological patterns, and functional outcome, in patients with GBS in Sri Lanka. Previous studies in Sri Lanka have described management aspects in an ICU setting. ${ }^{26}$ and treatment of GBS patients with modified plasma exchange. ${ }^{27}$ The third study looked at clinical features and outcome, but was only based on data from retrospective case record evaluation with its inherent drawbacks, and did not have neurophysiological confirmation. ${ }^{28}$

This oration is based on unpublished data from two prospective studies designed to describe the demographic, clinical and neurophysiological characteristics, and the functional outcome at discharge, in patients with Guillain-Barre syndrome in Sri Lanka. 
Study 1 - Guillain-Barre syndrome - a hospital based study*

Study 2 - Guillain-Barre syndrome - a clinical and neurophysiological study*

Both were hospital based studies conducted at the Institute of Neurology, National Hospital of Sri Lanka, Colombo (NHSL). Both prospectively evaluated patients with GBS using similar methodology.

One limitation of the first study (Study 1) was the lack of sensory conduction data on neurophysiological evaluation, due to technical problems encountered during the study period (lack of proper electrodes, etc.). Sensory conduction findings are important to distinguish AMAN from AMSAN, which may have a bearing on prognosis. The second study (Study 2) was designed to overcome this difficulty, with detailed neurophysiological evaluation by a consultant neurophysiologist at the neurophysiology unit of the NHSL.

This oration will summarise the results of these two studies, which taken together, will provide data regarding 117 patients with Guillain-Barre syndrome, the largest body of data on GBS from Sri Lanka to date.

\section{Purpose}

The objectives of these two studies were;

1. to describe the demographic and clinical characteristics

2. to determine the neurophysiological subtypes, and

3. to assess the functional outcome at discharge from hospital

in a population of Sri Lankan patients with GuillainBarre syndrome.

\section{Methodology}

Study 1 was conducted over a two year period, from January 1998 to December 1999, and studied 62 patients with GBS. Prior ethical approval for the study was obtained from the Ethical Review Committee of the Faculty of Medicine, Colombo.

Study 2 was also conducted over a two year period, from January 2002 to December 2003, and studied 55 patients with GBS. Prior ethical approval for the study was obtained from the Ethics Review Committee of the Sri Lanka Medical Association.

Both studies employed similar methodology. All consecutive patients with acute flaccid weakness admitted to a Neurology unit of the National Hospital of Sri Lanka, Colombo during the study period and providing informed consent were considered for enrolment. The entry criteria (see table 1) were based on accepted diagnostic criteria for Guillain-Barre syndrome..$^{19,29}$ Data were collected by medical officers, recorded on pre-validated, standardised data record forms, and entered into a database. Antecedent events during the 4 weeks prior to symptom onset were noted. Muscle power was graded from 0 to 5 according to the Medical Research Council (MRC) scale. Disability was graded from 0 to 6 (0- no symptoms, 6-dead) according to a widely used functional scale (see table 2 ), modified from Hughes et al. ${ }^{1,30}$ Presence of unexplained fluctuations in heart rate or blood pressure requiring treatment was considered as significant autonomic involvement.

Table 1. Entry criteria

\section{Inclusion criteria}

Progressive motor weakness of more than one limb

Hyporeflexia or areflexia

Relative symmetry

Progression up to a maximum of 4 weeks

Mild sensory symptoms or signs

Absence of fever at onset

\section{Exclusion criteria}

Pronounced or persistent asymmetry of weakness Sensory level

Other identifiable cause such as poliomyelitis, botulism, hysterical neuropathy, porphyria, diphtheria

Table 2. Disability scale
0 - no symptoms
1 - minor symptoms or signs
2 - able to walk $5 \mathrm{~m}$ without assistance
3 - able to walk $5 \mathrm{~m}$ with assistance or stick
4 - chair or bed bound
5 - requiring assisted ventilation
6 - dead

All patients had neurophysiological evaluation of the neuropathy during the hospital stay using accepted criteria. ${ }^{9,15}$ Cases were classified as acute inflammatory demyelinating polyradiculoneuropathy (AIDP) when evidence of demyelination was present in at least two limbs. Electrophysiological findings considered as indicative of demyelination were prolongation of distal latency, reduction of motor conduction velocity, and/ or prolongation of $\mathrm{F}$ wave latency. Cases were classified 
as axonal GBS when compound motor action potential (CMAP) amplitudes were diminished, in the absence of evidence of demyelination. In Study 2, patients with axonal GBS were further subdivided into acute motorsensory axonal neuropathy (AMSAN) or acute motor axonal neuropathy (AMAN), depending on the presence of sensory involvement. Patients in whom the electrophysiological features were insufficient to confirm demyelination or axonal degeneration were considered as 'unclassifiable'. Routine needle electromyographic (EMG) studies were not done. Cerebrospinal fluid (CSF) analysis was done as part of routine diagnostic work up. Neurophysiological assessment and CSF analysis are standard investigations in GBS. No additional invasive procedures were carried out for the purpose of the studies.

Treatment with specific immuno-modulatory therapy (plasma exchange or intravenous immunoglobulin), and other ancillary treatments were carried out according to clinical need. The studies did not influence the management of patients.

Outcome measures studied were peak motor deficit, peak functional disability, time taken to reach peak disability (time to nadir), need for intensive care, need for mechanical ventilation, duration of stay in the intensive care unit (ICU), duration of mechanical ventilation, death, and disability at discharge.

The demographic, clinical and neurophysiological findings and functional outcome between the two studies were compared using Student's t test, chi square test or Fisher's exact tests. Functional outcome between the AIDP and axonal GBS groups was also compared.

\section{Results}

\section{Demographic characteristics}

These two studies provide data regarding 117 patients with GBS (Study 1- 62 patients, Study 2 - 55 patients). The demographic features were broadly similar in the two study groups. Two-thirds were males (66.1\% in Study 1, 67.3\% in Study 2). Age distribution was similar, with young adults being predominantly affected. The mean age in Study 1 was $41.6( \pm 18.9)$ years, and in Study 2 was $38.0( \pm 17.17)$ years.

A history of antecedent infection in the preceding 4 weeks was common $(59.7 \%$ in Study $1,58.2 \%$ in Study 2), and this was usually a respiratory infection (54.1\% and $53.1 \%$ respectively, out of those with antecedent illness).

A preceding diarrhoeal illness was noted in only a few patients (12.9\% and $9.4 \%)$. There were no cases related to surgery, vaccination or pregnancy. No definite seasonal variation was observed in the admissions.

\section{Clinical characteristics (Table 3)}

The majority (66.1\% in Study 1, 60.9\% in Study 2) was admitted within seven days of symptom onset, and a further $25.8 \%$ and $30.9 \%$ respectively presented during the second week of illness.

All patients had limb weakness and hypo/areflexia as they were criteria for inclusion. The illness started with motor symptoms alone in most patients $(46.8 \%$ and $36.4 \%$ respectively), and lower limb involvement was common at onset (87.1\% and $61.8 \%)$. The maximal recorded weakness was severe (power MRC grade $0-2$ ) in most patients (56.5\% and $47.3 \%)$. It was mainly noted in the proximal musculature $(40.3 \%$ and $52.7 \%)$. Sensory symptoms were commonly noted (51.6\% and $65.5 \%)$, with objective sensory signs being unusual (14.5\% and $21.8 \%)$. Cranial nerve involvement was seen in the majority of patients (50\% and $72.7 \%)$, with bilateral facial weakness being the commonest (43.6\% and 54.5\%), and external ophthalmoplegia being rare ( 2 and 6 patients respectively). Significant autonomic involvement (considered as unexplained tachycardia or fluctuating blood pressure) was observed in many patients (29\% and 51\%).

\section{Investigations (Table 4)}

All patients had neurophysiological evaluation, except one patient in Study 2 who died before assessment. The findings were remarkably similar in the two studies. Acute inflammatory demyelinating polyradiculoneuropathy (AIDP) was the commonest neurophysiological subtype, seen in 42 patients $(67.8 \%)$ in Study 1, and in 37 patients (67.3\%) in Study 2. However, we also observed an unusually high prevalence of axonal degeneration (axonal GBS), seen in 15 patients (24.2\%) in Study 1, and in 16 patients (29.1\%) in Study 2. Patients with axonal GBS in Study 2 were further subdivided according to sensory findings. Almost all of them were of the acute motor axonal neuropathy (AMAN) type (14 out of 16 patients). Acute motorsensory axonal neuropathy (AMSAN) was rare. Neurophysiological findings were considered as unclassifiable in 5 patients in Study 1, and in one patient in Study 2.

Cerebrospinal fluid (CSF) analysis was done in the acute phase of the illness. CSF protein was elevated $(>0.4 \mathrm{~g} / \mathrm{l})$ in most patients $(67.8 \%$ in Study 1 , and $69.1 \%$ in Study 2), as expected, with levels being more than $1.0 \mathrm{~g} / \mathrm{l}$ in a large number (30.7\% and $43.6 \%)$.

\section{Treatment given}

The majority of patients $(87.5 \%$ in Study 1 , and 
Table 3. Clinical characteristics

\begin{tabular}{|c|c|c|c|c|c|c|}
\hline & \multicolumn{2}{|c|}{$\begin{array}{l}\text { Study } 1 \\
(n=62)\end{array}$} & \multicolumn{2}{|c|}{$\begin{array}{c}\text { Study } 2 \\
(n=55)\end{array}$} & \multicolumn{2}{|c|}{$\begin{array}{c}\text { Total } \\
(n=117)\end{array}$} \\
\hline & no. & $\%$ & no. & $\%$ & no & $\%$ \\
\hline Limb weakness & 62 & 100 & 55 & 100 & 117 & 100 \\
\hline Hypo/areflexia & 62 & 100 & 55 & 100 & 117 & 100 \\
\hline Onset with motor symptoms & 29 & 46.8 & 20 & 36.4 & 49 & 41.9 \\
\hline Onset with sensory symptoms & 19 & 30.7 & 19 & 34.5 & 38 & 32.5 \\
\hline Onset - both sensory \& motor symptoms & 14 & 22.6 & 16 & 29.1 & 30 & 25.6 \\
\hline Onset in lower limbs & 54 & 87.1 & 34 & 61.8 & 88 & 75.2 \\
\hline Onset in upper limbs & 8 & 12.9 & 13 & 23.6 & 21 & 18.0 \\
\hline Maximal weakness proximal & 25 & 40.3 & 29 & 52.7 & 54 & 46.2 \\
\hline Maximal weakness distal & 2 & 3.2 & 19 & 34.5 & 21 & 18.0 \\
\hline Maximal weakness global & 35 & 56.5 & 7 & 12.7 & 42 & 35.9 \\
\hline Sensory symptoms & 32 & 51.6 & 36 & 65.5 & 68 & 58.1 \\
\hline Sensory signs & 9 & 14.5 & 12 & 21.8 & 21 & 17.9 \\
\hline Cranial nerve involvement & 31 & 50.0 & 40 & 72.7 & 71 & 60.7 \\
\hline Facial weakness & 27 & 43.6 & 30 & 54.6 & 57 & 48.7 \\
\hline External ophthalmoplegia & 2 & 3.2 & 6 & 10.9 & 8 & 6.8 \\
\hline Bladder involvement & 14 & 22.8 & 7 & 12.7 & 21 & 18.0 \\
\hline Autonomic disturbances & 18 & 29.0 & 33 & 60.0 & 51 & 43.6 \\
\hline
\end{tabular}

Table 4. Investigations

\begin{tabular}{|c|c|c|c|c|c|c|}
\hline & \multicolumn{2}{|c|}{$\begin{array}{l}\text { Study } 1 \\
(n=62)\end{array}$} & \multicolumn{2}{|c|}{$\begin{array}{c}\text { Study } 2 \\
(n=55)\end{array}$} & \multicolumn{2}{|c|}{$\begin{array}{c}\text { Total } \\
(n=117)\end{array}$} \\
\hline & no. & $\%$ & no. & $\%$ & no & $\%$ \\
\hline \multicolumn{7}{|l|}{ Neurophysiology } \\
\hline Demyelination (AIDP) & 42 & 67.8 & 37 & 67.3 & 79 & 67.5 \\
\hline Axonal degeneration (axonal GBS) & 15 & 24.2 & 16 & 29.1 & 31 & 26.5 \\
\hline AMAN & - & - & 14 & 25.5 & - & - \\
\hline AMSAN & - & - & 2 & 3.6 & - & - \\
\hline Unclassifiable & 5 & 8.1 & 1 & 1.8 & 6 & 5.1 \\
\hline Not done & - & - & 1 & 1.8 & 1 & 0.9 \\
\hline \multicolumn{7}{|l|}{ Cerebrospinal fluid analysis } \\
\hline Elevated protein & 42 & 67.8 & 38 & 69.1 & 80 & 68.4 \\
\hline Cells $>5 / \mathrm{mcl}$ & 8 & 12.9 & 10 & 18.2 & 18 & 15.4 \\
\hline
\end{tabular}


$83.7 \%$ in Study 2) were given immuno-modulatory treatment, in addition to general medical care. This was mainly in the form of plasma exchange $(77.4 \%$ in Study 1, and $63.7 \%$ in Study 2). Out of them, most patients had only one exchange $(37.1 \%$ in Study 1 , and $48.6 \%$ in Study 2). Only a few patients received intravenous immunoglobulin (IVIg) therapy (6 in Study 1, and 19 in Study 2).

\section{Clinical course and functional outcome (Tables 5,6,7)}

The peak motor deficit was reached within 7 days in most patients (51.6\% in Study 1, and $43.6 \%$ in Study $2)$. The mean time to nadir was 8.45 days $( \pm 5.56)$ and 10.6 days $( \pm 10.6)$ respectively. Peak disability at nadir was severe (grade $\geq 4$ ) in the majority of patients $(67.7 \%$ in Study 1, and 80\% in Study 2), and was considered mild (grade $\leq 2$ ) in only 3 and 9 patients respectively.

A large number of patients required admission to an intensive care unit (ICU); 28 patients $(45.2 \%)$ in Study 1, and $24(43.6 \%)$ in Study 2. Respiratory involvement was the commonest cause for admission to the ICU. Mean duration of stay in the ICU was 20.6 $( \pm 14.6)$ days and $17.46( \pm 11.81)$ days, respectively. Respiratory involvement (vital capacity $<20 \mathrm{ml} / \mathrm{kg}$ ) was a common complication, seen in 29 patients $(46.8 \%)$ in Study 1, and in $23(41.8 \%)$ in Study 2. A large proportion of them required mechanical ventilation (27.4\% in Study $1,32.7 \%$ in Study 2$)$. Mean duration of ventilation was $15.1( \pm 13.9)$ days and $13.61( \pm 9.03)$ days, respectively.

Majority needed to be in hospital for more than two weeks (56.5\% in Study 1 and $43.6 \%$ in Study 2), with the mean duration of hospital stay at the NHSL being $21.6( \pm 13.4)$ days and $18.93( \pm 12.06)$ days. At the time of discharge, many patients had significant residual disability. A large number (40.7\% and 44\%) were chair-bound or bed-bound on discharge. Only a minority (17\% and $36 \%$ ) had mild disability, being able to walk home without assistance. Death (functional grade 6) occurred in 3 patients in Study 1, and 5 patients in Study 2, during the hospital stay.

\section{Comparison between Study 1 and Study 2}

The two studies were broadly similar in all the aspects studied; demographic features, main clinical features, neurophysiological types, CSF findings, and functional outcome (Tables 3,4,5,6, and 7). Statistically significant differences were noted only with regard to site of symptom onset (lower limbs commoner in Study $1, p=0.016)$, cranial nerve involvement (more in Study $2, p=0.012$ ), autonomic involvement (more in Study 2 , $\mathrm{p}=0.007$ ), number of patients with mild disability at peak of illness (peak disability $\leq$ grade 2 more in Study

Table 5. Clinical course and functional outcome

\begin{tabular}{|c|c|c|c|c|c|c|}
\hline & \multicolumn{2}{|c|}{$\begin{array}{c}\text { Study } 1 \\
(n=62)\end{array}$} & \multicolumn{2}{|c|}{$\begin{array}{c}\text { Study } 2 \\
(n=55)\end{array}$} & \multicolumn{2}{|c|}{$\begin{array}{c}\text { Total } \\
(n=117)\end{array}$} \\
\hline & no. & $\%$ & no. & $\%$ & no & $\%$ \\
\hline Peak disability $\geq$ grade 4 & 34 & 54.8 & 44 & 80 & 78 & 66.7 \\
\hline \multicolumn{7}{|l|}{ Time to peak motor deficit - } \\
\hline 1-7 days & 32 & 51.6 & 24 & 43.6 & 56 & 47.9 \\
\hline 8-14 days & 22 & 35.5 & 17 & 30.9 & 39 & 33.3 \\
\hline $15-21$ days & 5 & 8.1 & 8 & 14.5 & 13 & 11.1 \\
\hline more than 21 days & 3 & 4.8 & 6 & 10.9 & 9 & 7.7 \\
\hline Needed ICU care & 28 & 45.2 & 24 & 43.6 & 52 & 44.4 \\
\hline Respiratory involvement & 29 & 46.8 & 23 & 41.8 & 52 & 44.4 \\
\hline Needed assisted ventilation & 17 & 27.4 & 18 & 32.7 & 35 & 29.9 \\
\hline Duration of ventilation - & & $(n=17)$ & & $(n=18)$ & & $(n=35)$ \\
\hline 1-7 days & 4 & 23.5 & 5 & 27.8 & 9 & 25.7 \\
\hline 8-14 days & 9 & 52.9 & 6 & 23.3 & 15 & 42.9 \\
\hline $15-21$ days & 0 & 0 & 4 & 22.2 & 4 & 11.4 \\
\hline more than 21 days & 4 & 23.5 & 3 & 16.7 & 7 & 20.0 \\
\hline Death & 3 & 4.8 & 5 & 9.1 & 8 & 6.8 \\
\hline
\end{tabular}


Table 6. Outcome - Peak disability

\begin{tabular}{lrrrrrr}
\hline & \multicolumn{2}{c}{$\begin{array}{c}\text { Study 1 } \\
(n=62)\end{array}$} & \multicolumn{2}{c}{$\begin{array}{c}\text { Study 2 } \\
(n=55)\end{array}$} & \multicolumn{2}{c}{$\begin{array}{c}\text { Total } \\
(n=117)\end{array}$} \\
& $n o$. & $\%$ & $n o$. & $\%$ & $n o$ & $\%$ \\
\hline - no symptoms & 0 & 0 & 0 & 0 & 0 & 0 \\
1 - minor symptoms or signs & 0 & 0 & 1 & 1.8 & 1 & 0.9 \\
2 - able to walk 5 m without assistance & 3 & 4.8 & 8 & 14.5 & 11 & 9.4 \\
3 - able to walk 5 m with assistance & 17 & 27.4 & 2 & 3.6 & 19 & 16.2 \\
4 - chair or bed bound & 22 & 35.5 & 26 & 47.3 & 48 & 41.0 \\
5 - requiring assisted ventilation & 17 & 27.4 & 13 & 23.6 & 30 & 25.6 \\
6 - death & 3 & 4.8 & 5 & 9.1 & 8 & 6.8 \\
\hline
\end{tabular}

Table 7. Outcome - Disability at discharge

\begin{tabular}{lrrrrrr}
\hline & \multicolumn{2}{c}{$\begin{array}{c}\text { Study 1 } \\
(n=59)\end{array}$} & \multicolumn{2}{c}{$\begin{array}{c}\text { Study 2 } \\
(n=50)\end{array}$} & \multicolumn{2}{c}{$\begin{array}{c}\text { Total } \\
(n=109)\end{array}$} \\
& no. & $\%$ & no. & $\%$ & $n o$ & $\%$ \\
\hline - no symptoms & 0 & 0 & 0 & 0 & 0 & 0 \\
1 - minor symptoms or signs & 0 & 0 & 9 & 18 & 9 & 8.3 \\
2 - able to walk 5 m without assistance & 10 & 17.0 & 9 & 18 & 19 & 17.4 \\
3 - able to walk 5 m with assistance & 25 & 42.4 & 10 & 20 & 35 & 32.1 \\
4 - chair or bed bound & 24 & 40.7 & 22 & 44 & 46 & 42.2 \\
5 - requiring assisted ventilation & 0 & 0 & 0 & 0 & 0 & 0 \\
\hline
\end{tabular}

(8 patients died during hospital stay - 3 in Study 1, 5 in Study 2)

2, $p=0.0403$, and number of patients with mild disability at discharge (disability at discharge $\leq$ grade 2 more in Study 2, $p=0.233$ ). There were no statistically significant differences between the two studies in the other parameters studied.

\section{Functional outcome: AIDP vs. axonal GBS} (Table 8)

We compared the functional outcome in the two main neurophysiological subgroups, AIDP and axonal GBS. Outcome was noted to be poorer in patients with axonal GBS. In this group, hospital stays were longer $(p=0.0104), I C U$ stays were longer $(p=0.038)$, duration of ventilation was longer $(p=0.05$, of borderline significance), peak disability was more severe $(p=0.0128)$, and disability at discharge was more severe $(p=0.0044)$. Recovery was better (disability $\leq$ grade 2 on discharge) in the patients with AIDP ( $p=0.0058)$. There were no significant differences between the two groups in age distribution, sex distribution, time to nadir, and the number needing ICU care or ventilation.

\section{Discussion}

With the eradication of poliomyelitis being almost a reality, GBS has emerged as the commonest, and the most important, cause of acute flaccid paralysis. These two prospective studies conducted over a period of four years, taken together, provide a large collection 
Table 8. Functional outcome - AIDP vs. Axonal GBS

\begin{tabular}{|c|c|c|c|c|}
\hline & $\begin{array}{c}\text { AIDP } \\
\text { no. } \\
(n=79)\end{array}$ & $\begin{array}{c}\text { Axonal } \\
\text { GBS } \\
\text { no. } \\
(n=31)\end{array}$ & $X^{2}$ & $p$ \\
\hline Male sex & 54 & 18 & 1.04 & 0.3072 \\
\hline Time to peak disability $<1$ week & 27 & 9 & 0.27 & 0.6049 \\
\hline Peak disability $\geq$ grade 4 & 53 & 28 & 6.19 & 0.0128 \\
\hline Peak disability $\leq$ grade 2 & 11 & 1 & 2.62 & 0.1054 \\
\hline Needed ICU care & 34 & 15 & 0.26 & 0.6116 \\
\hline Needed assisted ventilation & 21 & 11 & 0.86 & 0.3551 \\
\hline ICU stay $>2$ weeks & 14 & 11 & 4.31 & 0.0380 \\
\hline Duration of ventilation $>2$ weeks & 4 & 6 & - & $0.0502^{*}$ \\
\hline Hospital stay $>2$ weeks & 40 & 24 & 6.57 & 0.0104 \\
\hline Disability at discharge $\geq$ grade 4 & 26 & 19 & 8.11 & 0.0044 \\
\hline Disability at discharge $\leq$ grade 2 & 25 & 2 & 7.6 & 0.0058 \\
\hline
\end{tabular}

* denotes $\mathrm{p}$ value obtained on Fisher's exact testing, where numbers were small

of data on 117 patients regarding this uncommon, yet serious, neurological illness. The two studies were comparable, as they were conducted in the same setting, over a similar duration, with identical inclusion and exclusion criteria, and using similar methodology. The results from the two studies were broadly similar, allowing these data to be collated and analysed together. This also highlights the similarity between the two patient populations, and therefore the validity and reproducibility of the data from the two studies.

Most series of GBS report a male preponderance. Only a slight excess of males (M:F ratio 0.8 to $1.3: 1$ ) has been reported from the Caucasian populations, $1,2,5,6,31-33$ while a relatively higher proportion (1.9 to 2.3:1) has been reported from the Asian countries..$^{7-9,16,34}$ Our finding of a male: female ratio of 2:1 was similar to the Asian figures. Interestingly, comparatively higher male prevalence rates have been noted with cases of AMAN, ${ }^{9,15}$ and the high prevalence in males reported from Asian series may be due to the higher frequency of axonal GBS.

Some studies have previously shown a bimodal age distribution with peaks occurring in young adults and elderly, ${ }^{6,31-33,35}$ or an increase with age with a peak in the elderly. $3,4,5,36,37$ The main exceptions to this have been the reports of GBS occurring predominantly in children from Northern China. ${ }^{10,15,16}$ In our series, the majority were young adults, as noted by Kaur et al and Dowling et al. ${ }^{7,33}$

An antecedent infection was noted in 59\% in our patients. This figure is similar to many series where such an event was recorded in $55-71.5 \%$ patients. This was usually a respiratory infection, similar to descriptions from the Western countries. ${ }^{31,37,38}$ Unlike reports from the Far Eastern countries, a preceding diarrhoeal illness was not a prominent feature in our study.

We did not find a seasonal preponderance, a finding seen in studies from European countries. ${ }^{3-5,31,36,39}$ Lack of a seasonal variation in GBS, in spite of its obvious association with infection, is attributed to the opposite seasonality of the antecedent respiratory and gastrointestinal infections. ${ }^{31}$ This is in contrast to the marked seasonal preponderance of GBS seen in summer epidemics of AMAN in Northern China, where it is associated with campylobacter enteritis. ${ }^{10,15-17}$ Clinical and laboratory features in our patients were characterised by prominent motor weakness with hyporeflexia or areflexia, common sensory symptoms but few objective sensory signs, cranial nerve involvement usually with bilateral facial weakness, and elevated CSF protein with few cells. These are similar to the previous descriptions of 'classical' GBS. ${ }^{1-9,33}$ 
Immuno-modulation by plasma exchange or intravenous immunoglobulin (IVIg) is the mainstay of therapy in GBS. The studies did not influence the decisions regarding institution of treatment or the type of treatment. Five plasma exchanges are usually recommended, but most of our patients received only one due to the logistic difficulties and the cost per procedure. IVIg, the most widely used treatment in the Western countries, was hardly used due to its prohibitive cost in Sri Lanka.

The clinical course of GBS in our patients is similar to what has been described previously. Assisted ventilation was required in $25.6 \%$, similar to the figures of $23-33 \%$ previously reported. $1,5,10,33,34$ The mean duration of ventilation was 15.1 days and 13.6 days in the two studies, similar to previous findings from elsewhere. . $^{2,934}$ The mean duration of hospital stay was 21.6 days and 18.9 days respectively, compared to a range of 21-66 days in other studies. ${ }^{3,5,7,9,29}$ Mean time to nadir was 8.45 days and 10.6 days in the two studies, compared to 5-9 days reported in the literature. $2,5,7,9,10$ Hospital mortality was $6.8 \%$, comparable to the mortality rates seen in most series (3.9-11.1\%).

The description of 'an axonal form of Guillain-Barre syndrome' in 1986, ${ }^{25}$ transformed the outlook on GBS and rekindled interest in its aetiology and pathological mechanisms, stimulating a large amount of new research. Hitherto considered a homogeneous clinical and pathological entity of an acute demyelinating neuropathy, it paved the way to the recognition of many cases of acute flaccid paralysis due to axonal neuropathy as of GBS, such as the summer outbreaks of AMAN from Northern China. ${ }^{10,15-23}$ AIDP was the neurophysiological type in $67.5 \%$ in our study, and $26.5 \%$ had axonal GBS. These figures lie in between the predominantly demyelinating picture seen in the Western countries, and the acute axonal forms seen in the Far East. Our rate of axonal GBS was much higher than the rates $(3-10 \%)$ described from the Western countries. ${ }^{2,11,12,6}$ However, we did not observe the predominance of axonal GBS over AIDP. Out of the patients with axonal GBS, the majority (14 out of 16) were of the AMAN type, similar to other Far Eastern data. Outcome in patients with axonal GBS is reported to be less favourable, when compared to AIDP. $12,23,24,25$ Our findings were similar, on comparing the pooled data on AIDP and axonal GBS from the two studies.

\section{Limitations}

Although originally planned, we were unable to perform sensory conduction studies in Study 1 due to technical problems encountered with the sensory recordings. Sensory studies are essential to enable proper classification of GBS, especially the axonal syndromes into acute motor or acute motor-sensory axonal neuropathies (AMAN, AMSAN). This limitation was overcome in the second study. The patients with axonal GBS in Study 2 were mostly of the AMAN type, and the AMSAN group was too small (2 out of 16) to enable any meaningful comparisons between these two groups.

We did not routinely investigate for an underlying aetiology in all patients, and in the cases investigated for an antecedent infection as part of the diagnostic work-up, the yield was low. Detailed investigations for aetiology should form a part of future studies, especially conditions such as Campylobacter jejuni infection, as the clinical features and the outcome may vary according to the underlying cause that triggers off GBS.

This was a hospital based study, and hospitalderived data cannot be readily extrapolated to a population at large. However, most patients with GBS seek admission to hospital due to the serious nature of the illness. The Institute of Neurology, NHSL is the final referral centre for neurological illness in Sri Lanka, and most patients with GBS are transferred there for tertiary care including plasma exchange. Furthermore, community based data are virtually impossible to obtain in GBS, due to its low incidence, and the need for hospital evaluation to establish the diagnosis. Almost all the reports in the literature are based on hospital derived data. Therefore, we believe these findings would be representative of the disease pattern in Sri Lanka.

\section{Conclusions}

Our results suggest that the pattern of clinical characteristics in Sri Lankan patients with GBS is similar to the classical picture of GBS described from other countries.

The neurophysiological characteristics lie in between the two ends of the spectrum represented by the West and the Far East, raising the possibility of an intermediate phenotype of GBS, which we can only speculate at. It may be interesting to observe whether a similar pattern exists in our neighbouring countries in South Asia, especially the Indian subcontinent. More research needs to be done to explore this possibility, as well as identify the aetiological factors and factors governing outcome in our population. We also need to look at the disease pattern and outcome in children, a group in which data on GBS are scanty. It is indeed surprising that only a handful of studies have been carried out on GBS in this country to date, considering the serious nature of the illness. A large multicentre study would be the way forward, to generate sufficient numbers needed to arrive at meaningful conclusions, especially regarding aetiology.

If our efforts to eradicate poliomyelitis from Sri Lanka are to succeed, we need to be able to rapidly and properly evaluate all patients with acute flaccid 
paralysis. GBS constitutes the majority of these patients. Facilities essential for diagnosis, such as neurophysiological assessments, are not available in most hospitals in the periphery where these patients would first present. Evaluation for underlying aetiological factors is vital, as recent studies have repeatedly shown the diversity in neuropathology and outcome in relation to aetiology. Facilities for aetiological diagnosis, even of the commonest responsible agents such as Campylobacter jejuni and common viruses, are not available even in the best of centres in Sri Lanka.

Treatment for GBS with plasma exchange or intravenous immunoglobulin is effective. However, these are available only in a handful of teaching hospitals in the country. Even where available, cost and logistic constraints preclude the delivery of ideal treatment (such as five plasma exchanges), as noted in our studies. Good supportive care is imperative in the acute phase, where ICU care and assisted ventilation can be life saving. Facilities for ICU care and ventilation are grossly inadequate in Sri Lanka, especially in the outstations. ${ }^{40}$ The lack of diagnostic and treatment facilities in the centres where these patients are first seen and acutely managed results in an influx of these patients to the National Hospital of Sri Lanka, Colombo (NHSL). This leads to unnecessary and avoidable delays in providing effective care which may adversely affect outcome, especially when requiring ventilation. This also entails enormous 'hidden' costs of transport, which we can ill afford.

IVIg is at least as equally effective as plasma exchange (PE). It is the preferred treatment in Western countries, especially considering its ease of use. The costs of the two treatments are considered to be similar, when one considers the cost of ancillary materials and skilled manpower needed in PE, which is a labour-intensive and time-intensive procedure. PE may not be a reality in all peripheral hospitals, due to its inherent need for prohibitively expensive equipment and technical expertise. However, provision of IVIg in the peripheral hospitals, at least in the main provincial and base hospitals, could possibly transform the outcome in our patients with GBS. If effective treatment can be administered early at the time and place of presentation, the need for ICU care, ventilation, and prolonged hospital stays, and transfers to the NHSL for PE can all be minimised.

GBS exacts a heavy toll on the patients, their families, and the health care delivery systems. The impact of disability on a young adult in his or her prime, with the resultant loss of earning capacity, sexual difficulties, effects on marital harmony, loss of self esteem, and psycho-social consequences, cannot be measured in mere economic terms. It is imperative that facilities for the effective management of this devastating illness are made readily available in all parts of the country.

\section{Acknowledgements}

I would like to thank the following: My collaborators in these two studies - Drs. Jagath Wijesekera, Sudath Gunasekera, Arjuna Fernando, Bimsara Senanayake, S. Puvanendiran, Buddhi Jayasekara, Tharangi Thilakaratne, Muditha Weerakkody, and Sandamali Premaratne. Prof. Rajitha Wickramasinghe, for providing statistical assistance. National Stroke Association of Sri Lanka, for partly supporting Drs. Buddhi Jayasekara and Tharangi Thilakaratne. Staff at the Institute of Neurology, National Hospital of Sri Lanka, Colombo and all the patients and individuals who participated in the studies.

\section{References}

1. Winer JB, Hughes RAC, Osmond C. A prospective study of acute idiopathic neuropathy. I. Clinical features and their prognostic value. Journal of Neurology, Neurosurgery \& Psychiatry 1988; 51: 605-12.

2. Ng KKP, Howard RS, Fish DR, et al. Management and outcome of severe Guillain-Barre syndrome. Quarterly Journal of Medicine 1995; 88: 243-50.

3. Kennedy RH, Danielson MA, Mulder DW, et al. Guillain-Barre syndrome: a 42-year epidemiologic and clinical study. Mayo Clinic Proceedings 1978; 53: 93-9.

4. Beghi E, Kurland LT, Mulder DW, et al. Guillain-Barre syndrome: clinicoepidemiologic features and effect of influenza vaccine. Archives of Neurology 1985; 42: 1053-7.

5. Hankey GJ. Guillain-Barre syndrome in Western Australia, 1980-1985. Medical Journal of Australia 1987; 146: 130-33.

6. Halls J, Brekjaer C, Friis ML. Guillain-Barre syndrome: diagnostic criteria, epidemiology, clinical course and prognosis. Acta Neurologiica Scandinavica 1988; 78: 118-22.

7. Kaur U, Chopra JS, Prabhakar S, et al. Guillain-Barre syndrome: a clinical, electrophysiological and biochemical study. Acta Neurologica Scandinavica 1986; 73: 394-402.

8. Samantray SK, Johnson SC, Mathai KV, et al. LandryGuillain-Barre-Strohl syndrome: a study of 302 cases. Medical Journal of Australia 1977; 2: 84-91.

9. Lyu RK, Tang LM, Cheng SY, et al. Guillain-Barre syndrome in Taiwan: a clinical study of 167 patients. Journal of Neurology, Neurosurgery and Psychiatry 1997; 63: 494-500.

10. McKhann GM, Cornblath DR, Griffin JW, et al. Acute motor axonal neuropathy: a frequent cause of acute flaccid paralysis in Northern China. Annals of Neurology 1993; 33 : 333-42.

11. Hadden RDM, Cornblath DR, Hughes RAC et al. Electrophysiological classification of Guillain-Barre syndrome: clinical associations and outcome. Annals of Neurology 1998; 44: 780-88. 
12. Rees JH, Soudain SE, Gregson NA, et al. Campylobacter jejuni infection and Guillain-Barre syndrome. New England Journal of Medicine 1995; 333: 1374-79.

13. Meulstee J, Van der Meche FGA, the Dutch Guillain-Barre Study Group. Electrodiagnostic criteria for polyneuropathy and demyelination: application in 135 patients with GuillainBarre syndrome. Journal of Neurology, Neurosurgery \& Psychiatry 1995; 59: 482-6.

14. The Italian Guillain-Barre Study Group. The prognosis and main prognostic indicators of Guillain-Barre syndrome: a multicentre prospective study of 297 patients. Brain 1996; 119: 2053-61.

15. Ho TW, Li CY, Gao CY, et al. Guillain-Barre syndrome in Northern China: relationship to Campylobacter jejuni infection and anti-glycolipid antibodies. Brain 1995; 118: 597-605.

16. McKhann GM, Cornblath DR, Ho T, et al. Clinical and electrophysiological aspects of acute paralytic disease of children and young adults in northern China. Lancet 1991; 338: 593-7.

17. Ho TW, Li CY, Cornblath DR, et al. Patterns of recovery in the Guillain-Barre syndromes. Neurology 1997; 48: 695-700.

18. Kuwabara S, Ogawara K, Koga M, et al. Hypereflexia in Guillain-Barre syndrome: relation with acute motor axonal neuropathy and anti-GM1 antibody. Journal of Neurology, Neurosurgery and Psychiatry 1995; 67: 180-84.

19. Sobue G, Li M, Aoki T, et al. Axonal pathology in Japanese Guillain-Barre syndrome: a study of 15 autopsied cases. Neurology 1997; 48: 1694-700.

20. Ho TW, Li CY, Ho TW, et al. Guillain-Barre syndrome in Northern China. The spectrum of neuropathological changes in clinically defined cases. Brain 1995; 118: 577-95.

21. Yuki N, Ho TW, Koga M, et al. Autoantibodies to GM1b and GalNac-GD1a: relationship to Campylobacter jejuni infection and acute motor axonal neuropathy in China. Journal of Neurological Sciences 1999; 164: 134-38.

22. Ogawara K, Kuwabara S, Mori M, et al. Axonal GuillainBarre syndrome: relationship to antiglycolipid antibodies and Campylobacter jejuni infection in Japan. Annals of Neurology 2000; 48: 624-31.

23. Chowdhury C, Arorsa A. Axonal Guillain-Barre syndrome: a review. Acta Neurologica Scandinavica 2001; 103 : 267-77.

24. Hadden RDM, Karch $\mathrm{H}$, Hartung $\mathrm{H}-\mathrm{P}$, et al. Preceding infections, immune factors and outcome in Guillain-Barre syndrome. Neurology 2001; 56: 758-65.

25. Feasby TE, Gilbert JJ, Brown WF, et al. An acute axonal form of Guillain-Barre neuropathy. Brain 1986; 109: 1115-26.
26. Gunawardana RH, Shanmuganathan PS, Ponniah S. Intensive care experience in Guillain-Barre syndrome. Ceylon Medical Journal 1992; 37: 85-87.

27. de Silva HJ, Gamage R, Herath HKN, et al. The treatment of Guillain-Barre syndrome by modified plasma exchange - a cost effective method for developing countries. Postgraduate Medical Journal 1987; 63: 1079-81.

28. Hewage C, Pathirana KD. Pattern of clinical features of Guillain-Barre syndrome in Southern Sri Lanka - a retrospective study. Galle Medical Journal 2002; 30-34.

29. Asbury AK, Cornblath DR. Assessment and current diagnostic criteria for Guillain-Barre syndrome. Annals of Neurology 1990; 27(suppl): S21-24.

30. Gregson NA, Koblar S, Hughes RAC. Antibodies to gangliosides in Guillain-Barre syndrome. Quarterly Journal of Medicine 1993; 86: 111-17.

31. Hughes RAC, Rees JH. Clinical and epidemiological features of Guillain-Barre syndrome. Journal of Infectious Diseases 1997; 176 (suppl 2): S92-S98.

32. Jiang GX, Cheng $\mathrm{Q}$, Link $\mathrm{H}$, et al. Epidemiological features of Guillain-Barre syndrome in Sweden 1978-93. Journal of Neurology, Neurosurgery \& Psychiatry 1997; 62: 447-53.

33. Dowling PC, Mennona JP, Cook SD. Guillain-Barre syndrome in New York. Journal of the American Medical Association 1977; 238: 317-8.

34. Baoxun Z, Yinchang $\mathrm{Y}$, Huifen $\mathrm{H}$, et al. Acute polyradiculitis (Guillain-Barre syndrome): an epidemiological study of 156 cases observed in Beijing. Annals of Neurology 1981; 9 (suppl): S146-S148.

35. Rees JH, Thompson RD, Smeeton NC, et al. Epidemiological study of Guillain-Barre syndrome in South East England. Journal of Neurology, Neurosurgery \& Psychiatry 1998; 64: 74-7.

36. Soffer D, Feldman S, Alter M. Epidemiology of Guillain-Barre syndrome. Neurology 1978; 28: 686-90.

37. Larsen JP, Kvale G, Nyland H. Epidemiology of Guillain-Barre syndrome in Norway. Acta Neurologica Scandinavica 1985; 71: $43-47$.

38. Winer JB, Hughes RAC, Osmond C. A prospective study of acute idiopathic neuropathy. II. Antecedent events. Journal of Neurology, Neurosurgery and Psychiatry 1988; 51: 613-18.

39. Hogg JE, Kobrin DE, Schoenberg BS. Guillain-Barre syndrome: Epidemiology and clinical features. Journal of Chronic Diseases 1979; 32: 227-31.

40. Yatawatte AB, Wanniarachchi CR, Goonasekera CDA. An audit of state sector intensive care services in Sri Lanka. Ceylon Medical Journal 2004; 49: 51-4. 